British Journal of Psychiatry (1995), 167, 549-554

\title{
Correspondence
}

Contents: Hypomania induced by gabapentin/ Chronic fatigue syndrome/Blu-Tack - a novel substance of abuse/Scale for assessing hedonic tone/ Neuroleptic associated extrapyramidal symptoms/ Case reports and confidentiality.

\section{Hypomania induced by gabapentin}

SIR: In response to the letter by Short et al (1995) we would like to raise a few issues which are pertinent to people with learning disabilities and refractory epilepsy.

The relationship between the management of epilepsy and the development of psychosis in a patient like this can be very complex. Short et al offered only one possible explanation for the occurrence of psychosis in a patient with learning disabilities with epilepsy. The interaction between epilepsy, use of anticonvulsants and the appearance of psychosis is explored by Trimble (1985). He states "although a few authors have investigated this in a systematic way, the role of anticonvulsant drugs in provoking inter-ictal chronic psychosis of epilepsy seems minimal". His paper was, of course, published before newer anticonvulsants were introduced. However, his classification of the psychosis of epilepsy into peri-ictal and inter-ictal psychosis is still useful.

In the latter group, also characterised as "chronic" (whereas the peri-ictal phenomena are described as "episodic"), he identifies "forced normalisation" as a form of inter-ictal psychosis. The term "forced normalisation" was introduced by Landolt (1958) describing cases in which an alternative clinical picture, namely psychosis or seizures, became overt depending on the EEG picture recorded during these episodes. Thus, during a psychosis, epileptic patients tended to lose their EEG abnormalities, which returned when the psychosis resolved. It is possible that the patient described by Short et al, could belong to this group. It is also unclear from their letter whether a good control of seizures was established with the use of gabapentin in this patient.

If good seizure control is to be achieved, the psychosis may become apparent and may need treatment in its own right. It could be argued that it is thus theoretically possible to precipitate a psychosis in some predisposed individuals by adequately treating their seizures. In learning disabilities it is of course difficult to obtain repeat EEGs on patients; however, in cases which have shown similar clinical features to the case reported by Short $e t$ al, it has been our practice to treat both the epilepsy and psychosis fully, thus seeking to enhance the patient's quality of life. As in every other case of epilepsy, not only the use of medication but the social and environmental measures play a vital part in the management of such complex cases.

We acknowledge, however, that gabapentin may have a mood elevating effect and it will be interesting to find out from long-term studies the effect of the newer anti-epileptic drugs on mood states.

LANDOLT, H. (1958) Serial encephalographic investigations during psychotic episodes in epileptic patients during schizophrenic attacks. In Lectures on Epilepsy (ed. L. de Haast). London: Elsevier.

SHORT, C. \& CoOKE, L. (1995) Hypomania induced by gabapentin (letter). British Journal of Psychiatry, 166, 679-680.

TrimBLE, M. R. (ed.) (1985) The psychoses of epilepsy and their treatment. In Psychopharmacology of Epilepsy (Ch. 6). Chichester: Wiley.

A. Hauck

Leicester Frith Hospital

S. BHAUMIK

Leicester LE3 9QF

\section{Chronic fatigue syndrome}

SIR: There is an ongoing need to investigate the role of biopsychosocial factors in the development of chronic fatigue syndrome (CFS) and to properly evaluate efficacy of treatment. However, Shanks \& Ho-Yen (1995) failed on both accounts largely because of methodological problems. First, they investigated various infective agents with a view to determining their role in the development of CFS. They purport to find evidence of a precipitating infection in $58 \%$ of their patients. However, in order to elucidate the role of infective agents in the pathophysiology of CFS, longitudinal prospective studies are required. While Imboden et al (1961) 
found that delayed recovery at six weeks was associated with psychological vulnerability in patients who had had influenza, White et al (in press) found that only patients with glandular fever, but not other viral infections were associated with fatigue at six months follow up. Wessely et al (1995) in a large prospective study, found no evidence that common infections were related to the development of CFS in primary care.

Second, Shanks \& Ho-Yen claimed that counselling resulted in an $80 \%$ improvement rate. While this result is very impressive the authors failed to provide any data which supports their sweeping conclusion. As one of the defining characteristics of CFS (Sharpe et al, 1991) is a $50 \%$ reduction in activity levels it is difficult to see how $80 \%$ of the group studied could have improved in terms of their disability when they were given advice to live within their limits.

Imbodin, J., Canter, A. \& Ciuff, L. (1961) Convalescence from influenza: a study of the psychological and clinical determinants. Archives of Internal Medicine, 108, 115-121.

Shanks, M. F. \& Ho-YeN, D. O. (1995) A clinical study of chronic fatigue syndrome. British Journal of Psychiarry, 166, 798-801.

Sharpe, M., Archard, L.. Banatvala, J., et al (1991) Chronic fatigue syndrome: Guidelines for research. Journal of the Royal Society of Medicine. 84, 118-121.

Wesseley, S., Chalder, T.. Pawlikowska. T., et al (1995) Postinfectious fatigue: prospective study in primary care. Lancet. 345, 1333-1338

Whitr, P. D., Thomas, J. M., Amess, J. S., et al (1995) The existence of a fatigue syndrome after glandular fever. Psychological Medicine, in press.

King's College School of Medicine

T. Chalder

\& Institute of Psychiatry

London SE5 $8 A Z$

AUTHOR'S REPLY: Ms Chalder is correct in saying that our findings do not prove that the evidence of viral infection detected in over half our sample of patients was closely connected with the subsequent chronic fatigue syndrome. In fact we showed that CFS patients with and without associated emotional disorder were biologically similar over a range of viral and immunological measures. This confirmed previous studies which showed that such measures, while they might contribute operationally to clinical definition, have little diagnostic value in this population far less any established etiological significance. Our reference to 'precipitating' viral infection was meant in the sense of association with the onset of symptoms, rather than implying aetiology, which of course could be in either direction.

Counselling to remain within energy limits is not logically incompatible with progressive diminution of the symptoms of fatigue. The detailed findings related to psychiatric diagnosis were not included in our paper, but in this early onset population generalised anxiety and panic disorders were much more frequently identified than the chronic depressive states described in populations with a longer duration of fatigue, and constituted over half the case diagnoses.

Patients with more recent onset of symptoms and a different pattern of associated emotional disorder appear to have a better prognosis. Admittedly this finding is based on a global clinical severity scale and simple data about occupation, although at the time the study was planned, more sophisticated assessments of fatigue symptomatology had not been developed.

Royal Cornhill Hospital

M.F. SHanks Aberdeen $A B 92 Z H$

\section{Blu-Tack - a novel substance of abuse}

SIR: We wish to report a novel form of substance abuse, namely the smoking of the adhesive material Blu-Tack. Three male patients (aged 23, 27 and 32 years) detained on our semi-secure unit for the chronic mentally ill, all with the diagnoses of paranoid schizophrenia and substance abuse, were found to have been attempting this activity in the enforced absence of their preferred substances.

Two methods were employed. Both commenced with drying the Blu-Tack on a radiator for three weeks (or until the experimenter's patience expired). The resulting residue was then either crumbled and smoked with tobacco in a roll-up cigarette, or inhaled from the burning end of a match. One of the three informed us that he experienced a very slight "high" along with a peculiar sensation in his stomach (the latter may however be related to his longstanding somatic delusions), but denied any other effects. Another (the originator, claiming to have thought of the procedure himself) found it to have virtually no effect on him. The third also found it an unrewarding activity. No changes were observed in these patients' mental states or physical conditions as a result of this particular activity.

Neither the hospital pharmacy nor the Regional Poisons Centre had any information regarding this phenomenon, and there does not appear to be any reference to it in the medical literature. The only other report we have heard emanates from HM Prison Saughton (CPN Ian Wishart, personal communication). 